\title{
Subclass of Harmonic Univalent Functions Associated with Generalized Derivative Operator
}

\author{
Ebtisam Eljamal, M. Darus \\ School of Mathematical Sciences, University Kebangsaan Malaysia, Bangi Selangor Malaysia, Bangi, 43600 Malaysia \\ E-mail:ebtisameljamal@yahoo.com; maslina@ukm.my
}

\begin{abstract}
Using a generalized derivative operator, we introduce and study a new subclass of harmonic univalent functions. In the present paper, we obtain numerous sharp results including coefficient conditions, extreme points, convolution properties and convex combinations for the above class of harmonic univalent functions. The results obtained for the class reduce to the corresponding results for various well-known classes in the literature.
\end{abstract}

Keywords - Harmonic univalent functions; derivative operator; extreme points; distortion inequalities.

\section{INTRODUCTION}

A continuous complex-valued function $f=u+i v$ is defined in a simply-connected complex domain $D$ is said to be harmonic in $D$ if both $u$ and $v$ are real harmonic in $D$. Such functions can be expressed as

$$
f=h+\bar{g}
$$

where $h$ and $g$ are analytic in $D$. We call $h$ the analytic part and $g$ the co-analytic part of $f$. A necessary and sufficient condition for $f$ to be locally univalent and sense-preserving in $D$ is that $|\bar{h}(z)|>|\bar{g}(z)|$ for all $z$ in $D$ (see 1$)$. Let $S_{H}$ be the class of functions of the form (1) that are harmonic univalent and sense-preserving in the unit disk $U\{z:|z|<$ 1 ) for which $f(0)=f_{z}(0)-1=0$. Then for $f=h+\bar{g} \in$ $S_{H}$ we may express the analytic functions $h$ and $g$ as

$$
\begin{array}{r}
h(z)=z+\sum_{k=2}^{\infty} a_{k} z^{k}, \\
g(z)=z+\sum_{k=1}^{\infty} b_{k} z^{k}, z \in U,\left|b_{1}\right|<1 .
\end{array}
$$

In 1984, Clunie and Sheil-Small [1] investigated the class $S_{H}$ as well as its geometric subclasses

and obtained some coefficient bounds. Since then, there have been several related papers on $S_{H}$ and its subclasses.

Let $\bar{S}_{H}$ denote the subclasses of $S_{H}$ consisting of harmonic functions of the form

$$
f_{m}=h+\bar{g}_{m}
$$

Where

$$
\begin{gathered}
h(z)=z+\sum_{k=2}^{\infty} a_{k} z^{k}, \\
g_{m}(z)=(-1)^{m} z+\sum_{k=1}^{\infty} b_{k} z^{k}, z \in U,\left|b_{1}\right|<1 .
\end{gathered}
$$

Now we will introduce generalized derivative operator for $f=h+\bar{g}$ given by (2). For fixed positive natural $m$ and $\lambda_{2} \geq \lambda_{1} \geq 0$

$D_{\lambda_{1}, \lambda_{2}}^{m, k} f(z)=D_{\lambda_{1}, \lambda_{2}}^{m, k} h(z)+(-1)^{m} D_{\lambda_{1}, \lambda_{2}}^{m, k} g(z), z \in U$

where

$$
D_{\lambda_{1}, \lambda_{2}}^{m, k} h(z)=z+\sum_{k=2}^{\infty}\left(\frac{1+\left(\lambda_{1}+\lambda_{2}\right)(k-1)}{1+\lambda_{2}(k-1)}\right)^{m} a_{k} z^{k}
$$

and

$$
D_{\lambda_{1}, \lambda_{2}}^{m, k} g(z)=\sum_{k=1}^{\infty}\left(\frac{1+\left(\lambda_{1}+\lambda_{2}\right)(k-1)}{1+\lambda_{2}(k-1)}\right)^{m} a_{k} z^{k}
$$

We note that by specializing the parameters, especially when $\lambda_{1}=\lambda_{2}=0, D_{\lambda_{1}, \lambda_{2}}^{m, k}$ reduces to $D^{m}$ which introduced by Salagean in [3]. Now we will introduce the following definition.

\section{Definition 1:}

The function $f=h+\bar{g}$ defined by (2) is in the class $S_{H}=\left(\ell, \alpha, m, \lambda_{1}, \lambda_{2}\right)$ if

$$
\operatorname{Re}\left(\frac{D_{\lambda_{1}, \lambda_{2}}^{m+1, k} f(z)}{D_{\lambda_{1}, \lambda_{2}}^{m, k} f(z)}\right) \geq \ell\left|\frac{D_{\lambda_{1}, \lambda_{2}}^{m+1, k} f(z)}{D_{\lambda_{1}, \lambda_{2}}^{m, k} f(z)}\right|+\alpha
$$


where

$0 \leq \ell<\infty, 0 \leq \alpha<1, m \in N \cup\{0\}, \lambda_{2} \geq \lambda_{1} \geq 0$.

Also let $\bar{S}_{H}\left(\ell, \alpha, m, \lambda_{1}, \lambda_{2}\right)=S_{H}\left(\ell, \alpha, m, \lambda_{1}, \lambda_{2}\right) \cap \bar{S}_{H}$.

We note that by special choices of $\ell, m, \lambda_{1}, \lambda_{2}$ we obtain the following classes studied by various authors:

i. $\bar{S}_{H}(0, \alpha, 0,0,0) \equiv H S(\alpha)$ is the class of sensepreserving harmonic univalent functions $f$ which are starlike of order $\alpha$ in $U$, where studied by Jahangiri [2].

ii. $\bar{S}_{H}(0, \alpha, 1,0,0) \equiv H K(\alpha)$ is the class of sensepreserving, harmonic univalent functions $f$ which are convex of order $\alpha$ in $U$, which studied by Jahangiri [2].

iii. $\quad \bar{S}_{H}(\ell, \alpha, m, 0,0) \equiv \bar{S}_{H}(\ell, \alpha, m)$ is the class of sensepreserving, harmonic univalent functions $f$ which are starlike of order $\alpha$ in $U$, studied by T.S. Joshi [4].

In the present paper we aim to investigate several properties of subclasses of harmonic functions. In particular the coefficient bound, distortion theorem and extreme points. In recent years many researchers have studied various subclasses of $S_{H}$ for example [2], [5], [6], [7] and [8] and. The most recent ones, can have a look at [9], [12]. In this paper, we extend some well-known results to the classes $S_{H}\left(\ell, \alpha, m, \lambda_{1}, \lambda_{2}\right)$ and $\bar{S}_{H}\left(\ell, \alpha, m, \lambda_{1}, \lambda_{2}\right)$.

\section{MAin Result}

In our first theorem, we introduce a sufficient coefficient bound for harmonic functions in $S_{H}\left(\ell, \alpha, m, \lambda_{1}, \lambda_{2}\right)$

\section{Theorem 1:}

Let $f=h+\bar{g}$ given by (2). Furthermore, let.

$\sum_{k=1}^{\infty}\left[\frac{\psi^{m}[\psi(1+\ell)-\ell-\alpha]}{1-\alpha}\left|a_{k}\right|+\frac{\psi^{m}[\psi(1+\ell)+\ell+\alpha]}{1-\alpha}\left|b_{k}\right|\right] \leq 2$

where $\psi^{m}=\left[\frac{1+\left(\lambda_{1}+\lambda_{2}\right)(k-1)}{1+\lambda_{2}(k-1)}\right]^{m}, a_{1}=1,0 \leq \alpha<1$, $0 \leq \ell<\infty, m \in N \cup\{0\}$ and $\lambda_{2} \geq \lambda_{1} \geq 0$, then $f \in S_{H}\left(\ell, \alpha, m, \lambda_{1}, \lambda_{2}\right)$.

Proof: According to (3) and (4) we have

$$
\operatorname{Re}\left(\frac{D_{\lambda_{1}, \lambda_{2}}^{m+1, k} f(z)}{D_{\lambda_{1}, \lambda_{2}}^{m, k} f(z)}\right) \geq \ell\left|\frac{D_{\lambda_{1}, \lambda_{2}}^{m+1, k} f(z)}{D_{\lambda_{1}, \lambda_{2}}^{m, k} f(z)}-1\right|+\alpha .
$$

This is equivalent to $\operatorname{Re}\left(\frac{A(z)}{B(z)}\right)>\alpha$ where $A(z)=$ $(1+\ell) D_{\lambda_{1}, \lambda_{2}}^{m+1, k} f(z)-\ell D_{\lambda_{1}, \lambda_{2}}^{m+1, k} f(z)$ and

$B(z)=D_{\lambda_{1}, \lambda_{2}}^{m, k} f(z)$. Using the fact that, $\operatorname{Re}(w)>\alpha$ if $|1-\alpha+\mathrm{w}| \geq|1+\alpha-\mathrm{w}|$, it suffices to show that

$$
|A(z)+(1-\alpha) \mathrm{B}(\mathrm{z})| \geq|A(z)-(1+\alpha) \mathrm{B}(\mathrm{z})|
$$

substituting values of $A(z)$ and $B(z)$ and with simple calculations we led to

$$
\mid(2-\alpha) z+\sum_{k=2}^{\infty} \psi^{m}[(1+\ell) \psi-\ell+(1-\alpha)] a_{k} z^{k}
$$

$$
\begin{array}{r}
-(-1)^{m} \sum_{k=1}^{\infty} \psi^{m}[(1+\ell) \psi-\ell-(1-\alpha)] \bar{b}_{k} \bar{z}^{k} \mid \\
-\mid \alpha z+\sum_{k=2}^{\infty} \psi^{m}[(1+\ell) \psi-\ell+(1-\alpha)] a_{k} z^{k}+ \\
(-1)^{m} \sum_{k=1}^{\infty} \psi^{m}[(1+\ell) \psi-\ell-(1-\alpha)] \bar{b}_{k} \bar{z}^{k} \mid \\
\left.\geq 2(1-\alpha)|z|-\sum_{k=2}^{\infty} \psi^{m}[2(1+\ell) \psi-2 \ell-2 \alpha)\right] \\
\left|a_{k}\right|\left|z^{k}\right|-(-1)^{m} \sum_{k=1}^{\infty} \psi^{m}[2(1+\ell) \psi+2 \ell+ \\
2 \alpha)]\left|a_{k}\right|\left|z^{k-1}\right|, \\
\geq 2(1-\alpha)|z|\left\{1-\sum_{k=2}^{\infty} \psi^{m}\left[\frac{(1+\ell) \psi-\ell-\alpha}{1-\alpha}\right]\right. \\
\left|a_{k}\right|\left|z^{k-1}\right|-(-1)^{m} \sum_{k=2}^{\infty} \psi^{m}\left[\frac{(1+\ell) \psi+\ell+\alpha}{1-\alpha}\right] \\
\left.\bar{b}_{k}\left|\bar{z}^{k}\right|\right\}
\end{array}
$$

by assumption. Hence the proof is complete.

The harmonic univalent functions

where

$$
\begin{gathered}
f(z)=z+\sum_{k=2}^{\infty} \frac{(1-\alpha)}{\psi^{m}[(1+\ell) \psi-\ell-\alpha} x_{k} z^{k}+ \\
\sum_{k=1}^{\infty} \frac{(1-\alpha)}{\psi^{m}[(1+\ell) \psi+\ell+\alpha} \overline{y z}^{k}
\end{gathered}
$$

$$
\sum_{k=2}^{\infty}\left|x_{k}\right|+\sum_{k=1}^{\infty}\left|y_{k}\right|=1
$$

shows that the coefficient bound given by (6) is sharp.

\section{Theorem 2:}

Let $f_{m}=h+\bar{g}_{m}$ be given by (3).

Then $f_{m} \in \bar{S}_{H}\left(\ell, \alpha, m, \lambda_{1}, \lambda_{2}\right)$ if and only if

$$
\begin{aligned}
& \sum_{k=1}^{\infty}\left[\frac{\psi^{\mathrm{m}}[(1+\ell) \psi-\ell-\alpha]}{1-\alpha}\right]\left|a_{k}\right|+ \\
& {\left[\frac{\psi^{\mathrm{m}}[(1+\ell) \psi+\ell+\alpha]}{1-\alpha}\right]\left|b_{k}\right| \leq 2}
\end{aligned}
$$

where $\psi^{m}=\left[\frac{1+\left(\lambda_{1}+\lambda_{2}\right)(k-1)}{1+\lambda_{2}(k-1)}\right]^{m}, a_{1}=1,0 \leq \alpha<1,0 \leq \ell<$ $\infty, m \in N \cup\{0\}$ and $\lambda_{2} \geq \lambda_{1} \geq 0$.

Proof: Since $\bar{S}_{H}\left(\ell, \alpha, m, \lambda_{1}, \lambda_{2}\right) \subset \bar{S}_{H}\left(\ell, \alpha, m, \lambda_{1}\right.$,

$\lambda_{2}$ ). we only need to prove the "only if" part of the theorem. Note that a necessary and sufficient condition for $f_{m}=h+$ $\bar{g}_{m}$ given by (3) to be in $\bar{S}_{H}\left(\ell, \alpha, m, \lambda_{1}, \lambda_{2}\right)$ is that

$$
\operatorname{Re}\left(\frac{D_{\lambda_{1}, \lambda_{2}}^{m+1, k} f(z)}{D_{\lambda_{1}, \lambda_{2}}^{m, k} f(z)}\right) \geq \ell\left|\frac{D_{\lambda_{1}, \lambda_{2}}^{m+1, k} f(z)}{D_{\lambda_{1}, \lambda_{2}}^{m, k} f(z)}-1\right|+\alpha
$$

which is equivalent to

$$
\operatorname{Re}\left\{\frac{(1-\alpha) D_{\lambda_{1}, \lambda_{2}}^{m, k} f_{m}+(\ell+\alpha) D_{\lambda_{1}, \lambda_{2}}^{m, k} f_{m}}{D_{\lambda_{1}, \lambda_{2}}^{m, k} f_{m}}\right\}
$$




$$
\begin{aligned}
= & \operatorname{Re}\left\{\frac{(1-\alpha) z-\sum_{k=2}^{\infty} \psi^{m}[(1+\ell) \psi-\ell-\alpha] a_{k} z^{k}}{z-\sum_{k=2}^{\infty} \psi^{m} a_{k} z^{k}+(-1)^{2 m} \sum_{k=1}^{\infty} \psi^{m} \bar{b}_{k} \bar{z}^{k}}\right. \\
& \left.+\frac{(-1)^{2 m-1} \sum_{k=1}^{\infty} \psi^{m}[(1+\ell) \psi+\ell+\alpha] \bar{b}_{k} \bar{z}^{k}}{z-\sum_{k=2}^{\infty} \psi^{m} a_{k} z^{k}+(-1)^{2 m} \sum_{k=1}^{\infty} \psi^{m} \bar{b}_{k} \bar{z}^{k}}\right\} \geq 0
\end{aligned}
$$

The above condition must holds for all values of $z,|z|=r<$

1. Choosing $z$ on the positive real axis where $0 \leq z=r<$ 1. We have

$$
\begin{aligned}
& \left\{\frac{(1-\alpha)-\sum_{k=2}^{\infty} \psi^{m}[(1+\ell) \psi-\ell-\alpha] a_{k} r^{k-1}}{1-\sum_{k=2}^{\infty} \psi^{m} a_{k} r^{k-1}+(-1)^{2 m} \sum_{k=1}^{\infty} \psi^{m} b_{k} \bar{r}^{k-1}}+\right. \\
& \left.\frac{(-1)^{2 m-1} \sum_{k=1}^{\infty} \psi^{m}[(1+\ell) \psi+\ell+\alpha] b_{k} \bar{r}^{k-1}}{1-\sum_{k=2}^{\infty} \psi^{m} a_{k} r^{k-1}+(-1)^{2 m} \sum_{k=1}^{\infty} \psi^{m} b_{k} \bar{r}^{k-1}}\right\} \geq 0 .
\end{aligned}
$$

If the condition (7) does not hold, then the numerator in (8) is negative for $r$ sufficiently close to 1 . Thus there exists $z_{0}=r_{0}$ in $(0,1)$ for which the quotient in (8) is negative. This contradicts the required condition for $f_{m} \in$ $\bar{S}_{H}\left(\ell, \alpha, m, \lambda_{1}, \lambda_{2}\right)$ and so the proof is complete.

\section{Theorem 3:}

Let $f_{m}$ be given by (3). Then $f_{m} \in \bar{S}_{H}\left(\ell, \alpha, m, \lambda_{1}, \lambda_{2}\right)$ if and only if

$$
f_{m}(z)=\sum_{k=1}^{\infty}\left(x_{k} h_{k}(z)+y_{k} g_{m k}(z)\right)
$$

where

$$
\begin{gathered}
h_{1}(z)=z, h_{k}(z)= \\
z-\frac{1-\alpha}{\psi^{m}[(1+\ell) \psi-\ell-\alpha]} z^{k}, \quad k=2,3, \ldots
\end{gathered}
$$

$g_{m k}(z)=$

$z+(-1)^{m-1} \frac{1-\alpha}{\psi^{m}[(1+\ell) \psi+\ell+\alpha]} z^{k}, k=2,3, \ldots$

and

$$
x_{m} \geq 0, y_{m} \geq 0, x_{1}=1-\sum_{k=2}^{\infty}\left(x_{m}+y_{m}\right) \geq 0 .
$$

In particular, the extreme points of $\bar{S}_{H}\left(\ell, \alpha, m, \lambda_{1}, \lambda_{2}\right)$ are $\left\{h_{m}\right\}$ and $\left\{g_{m k}\right\}$.

\section{Proof: Let}

$$
\begin{gathered}
f_{m}(z)=\sum_{k=1}^{\infty}\left(x_{k} h_{k}(z)+y_{k} g_{m k}(z)\right) \\
=\sum_{k=1}^{\infty}\left(x_{m}+y_{m}\right) z-\sum_{k=2}^{\infty} \frac{1-\alpha}{\psi^{m}[(1+\ell) \psi-\ell-\alpha]} x_{k} z^{k}+ \\
(-1)^{m-1} \sum_{k=1}^{\infty} \frac{1-\alpha}{\psi^{m}[(1+\ell) \psi+\ell+\alpha]} y_{k} \bar{z}^{k} .
\end{gathered}
$$

Then

$$
\begin{aligned}
\sum_{k=2}^{\infty} \frac{\psi^{m}[(1+\ell) \psi-\ell-\alpha]}{1-\alpha} & \left|a_{k}\right|+\sum_{k=1}^{\infty} \frac{\psi^{m}[(1+\ell) \psi+\ell+\alpha]}{1-\alpha}\left|b_{k}\right| \\
= & \sum_{k=2}^{\infty} x_{k}+\sum_{k=1}^{\infty} y_{k}=1-x_{1} \leq 1
\end{aligned}
$$

and so $f_{m} \in \bar{S}_{H}\left(\ell, \alpha, m, \lambda_{1}, \lambda_{2}\right)$.

Conversely, suppose that $f_{m} \in \bar{S}_{H}\left(\ell, \alpha, m, \lambda_{1}, \lambda_{2}\right)$ setting

$$
\begin{array}{ll}
x_{k}=\frac{\psi^{m}[(1+\ell) \psi-\ell-\alpha]}{1-\alpha} a_{k} \quad k=2,3, \ldots \\
y_{k}=\frac{\psi^{m}[(1+\ell) \psi+\ell+\alpha]}{1-\alpha} b_{k} \quad k=2,3, \ldots
\end{array}
$$

where $\sum_{k=1}^{\infty}\left(x_{k}+y_{k}\right)=1$, we obtain $f_{m}(z)=$ $\sum_{k=1}^{\infty}\left(x_{k} h_{k}(z)+y_{k} g_{m k}\right)$ as required.

\section{Theorem 4:}

The class $\bar{S}_{H}\left(\ell, \alpha, m, \lambda_{1}, \lambda_{2}\right)$ is closed under convex combination.

Proof: For $i=1,2, \ldots$, suppose $f_{m i} \in \bar{S}_{H}\left(\ell, \alpha, m, \lambda_{1}, \lambda_{2}\right)$ where

$$
f_{m i}=z-\sum_{k=2}^{\infty}\left|a_{i k}\right| z^{k}+(-1)^{m} \sum_{k=1}^{\infty}\left|b_{i k}\right| \bar{z}^{k}
$$

then by Theorem 2

$$
\sum_{k=2}^{\infty} \frac{\psi^{m}[(1+\ell) \psi-\ell-\alpha]}{1-\alpha}\left|a_{i k}\right|+\sum_{k=2}^{\infty} \frac{\psi^{m}[(1+\ell) \psi+\ell+\alpha]}{1-\alpha}\left|b_{i k}\right| \leq 1
$$

where $\quad \psi^{m}=\left[\frac{1+\left(\lambda_{1}+\lambda_{2}\right)(k-1)}{1+\lambda_{2}(k-1)}\right]^{m}$. For $\sum_{i=1}^{\infty} t_{i}=1,0 \leq t_{i} \leq 1$ the convex combination of $f_{m i}$ may be written as

$$
\begin{gathered}
\sum_{i=1}^{\infty} t_{i} f_{m i}(z)=z-\sum_{k=2}^{\infty}\left(\sum_{i=1}^{\infty} t_{i}\left|a_{i k}\right|\right) z^{k} \\
+(-1)^{m} \sum_{k=1}^{\infty}\left(\sum_{i=1}^{\infty} t_{i}\left|b_{i k}\right|\right) \bar{z}^{k}
\end{gathered}
$$

hence by (9)

$$
\begin{aligned}
& \sum_{k=2}^{\infty} \frac{\psi^{m}[(1+\ell) \psi-\ell-\alpha]}{1-\alpha}\left(\sum_{i=1}^{\infty} t_{i}\left|a_{i k}\right|\right) \\
& +\sum_{k=1}^{\infty} \frac{\psi^{m}[(1+\ell) \psi+\ell+\alpha]}{1-\alpha}\left(\sum_{i=1}^{\infty} t_{i}\left|b_{i k}\right|\right) \\
& =\sum_{i=1}^{\infty} t_{i}\left[\sum_{k=2}^{\infty} \frac{\psi^{m}[(1+\ell) \psi-\ell-\alpha]}{1-\alpha}\left|a_{i k}\right|\right. \\
& \left.+\sum_{k=1}^{\infty} \frac{\psi^{m}[(1+\ell) \psi+\ell+\alpha]}{1-\alpha}\left|b_{i k}\right|\right]
\end{aligned}
$$

and therefore $\sum_{i=1}^{\infty} t_{i} f_{m i} \in \bar{S}_{H}\left(\ell, \alpha, m, \lambda_{1}, \lambda_{2}\right)$. This completes the proof. 


\section{CONCLUSIONS}

In this paper we use a generalized derivative operator to introduce a new subclass of harmonic univalent function. In our first theorem we introduce a sufficient coefficient bound for harmonic functions in $\mathrm{S} \_\mathrm{H}\left(1, \alpha, \mathrm{m}, \lambda \_1, \lambda 2\right)$. In the second theorem, it is shown that the condition (6) is also necessary for functions $\mathrm{f} \mathrm{m}=\mathrm{h}+{ }^{-} \mathrm{g}$, where $\mathrm{h}$ and $\mathrm{g}$. $\mathrm{m}$ are of the form (2). In the third theorem, we determine the extreme points of closed convex hulls of $\left(1, \alpha, \mathrm{m}, \lambda \_1, \lambda \_2\right)$. In the last theorem we discuss the convex combinations class $(1, \alpha, \mathrm{m}$, $\lambda 1, \lambda 2$ ). Some other results can be discussed by using the new class introduced in this paper.

\section{ACKNOWLEDGMENT}

The work here was supported by UKM-ST-06FRGS0244-2010.

\section{REFERENCES}

[1] Clunie and T. Shell-Smail, Harmonic univalent functions, Ann. Acad. Sci. Fenn. Ser. A. I. Math, 9: (1984), 3-25.

[2] J. M. Jahangiri, Harmonic functions starlike in the unit disc, J. Math. Anal. Appl, bf 235:(1999), 470-477.
[3] G. S. Salagean, Subclass of univalent functions, Lecture Notes in Math.,Springer-Verlag, Berlin, Heidelberg and New York., 1013: (1983), 362-372.

[4] T. Scheil- Small, Subclasses of harmonic univalent functions associated with Salagean derivative, J. Math. Anal. Appl, $23(3)\}:(2007), 303--309$.

[5] O.P.Ahuja, R. Aghalary and S. B. Joshi, Harmonic univalent functions associated with k-uniformly starlike functions, Math Sci Res.J, 9(1): (2005), 9-17.

[6] S. Yalcin, On certain harmonic univalent functions defined by Salagean derivative, Soochow J.Math, 31(3): (2005), 321-331.

[7] J. M. Jahangiri, G. Murugusundaramoorthy and K. Vijaya, Salageantype harmonic univalent functions, South. J. Pure Appl. Math, 2 (2002), 77-82.

[8] H. Silverman and E. M. Silvia, Subclasses of harmonic univalent functions, New Zealand J. Math, 28: (1999), 275-284.

[9] K.Al-Shaqsi and M.Darus, On Harmonic Functions defined by derivative operator. Journal of Inequalities and Applications, 2008 (2008), Article ID 263413, 10 pages.

[10] K. Al-Shaqsi and M. Darus, On generalizations of convolution for harmonic functions, Far East J. Math. Sci. (FJMS) 33(3) (2009), 387-399.

[11] K. Al-Shaqsi and M.Darus, On certain class of harmonic univalent functions. Int. J. Contemp. Math Sci, 4(24) (2009),1193-1207.

[12] K. Al-Shaqsi, M. Darus and O. A. Fadipe-Joseph, A new subclass of Salagean-Type harmonic Univalent Functions, Abstract and Applied Analysis, 2010 (2010). Article ID 821531, 12 pages. 